\title{
Model Matching Ho State Feedback Controller Design using Linear Matrix Inequality Optimization Method for Obtaining Negative Stiffness on 4-Pole U-type Hybrid Electromagnet
}

\author{
Barış Can Yalçın ${ }^{1}$, Kadir Erkan ${ }^{2}$
}

\begin{abstract}
Pole U-type hybrid electromagnetic levitation systems can levitate ferromagnetic materials not only using the force of electromagnetic parts but also using the force of permanent magnet parts. This specification gives an opportunity to use 4-Pole U-type hybrid electromagnetic levitation systems for obtaining negative stiffness in zero-power control applications. However, the system shows highly non-linear behavior and obtaining negative stiffness for vibration isolation purpose becomes a control problem that has to be optimized in terms of non-linearity. The objective of this paper is to present a model matching $\mathrm{H} \infty$ state feedback controller using linear matrix inequality optimization method for obtaining negative stiffness on 4-Pole U-type hybrid electromagnet. Even though the subject of negative stiffness is always attached to the subject of zeropower control in literature, this work only focuses negative stiffness part. The effectiveness of proposed optimal control method is proven by simulation results.
\end{abstract}

Keywords - negative stiffness, infinity stiffness, linear matrix inequality, maglev.

\section{INTRODUCTION}

Usage of permanent magnets in the structure of electromagnets constructing to hybrid design brings very important advantages such as decreased volume and a more compact design. Moreover, necessary force for levitation of ferromagnetic material can be supplied by only the force of permanent magnet(s). That's why hybrid electromagnets are so popular in the field of zero-power control [1-5]. Using hybrid electromagnets, magnetic levitation can be achieved with no energy consumption between pre-determined boundaries. However, the system's behavior needs to be stabilized.

During last few decades, U-Type electromagnets have been popularly used in many industrial applications that include suspending ferromagnetic objects. However, controlling more than one degree of freedom cannot be possible using a standard U-Type electromagnet. To get rid of this problem, 4pole u-type electromagnet structure has been proposed [6, 7].

Barış Can Yalçın ${ }^{1}$, Kadir Erkan ${ }^{2,}$ Yildiz Technical University, Turkey
Hence, control capability in multi-degree of freedom and full redundancy are obtained. Yet, in this work, levitation is realized only for one degree of freedom due to the constraints that are brought by the aim of obtaining negative stiffness.

One of the most important industrial application area of electromagnets is vibration isolation [2-4, 7-9]. In the vibration isolation perspective, there are two types of vibrations that have to be focused before starting a vibration isolation system [10]. First type is direct disturbance; it occurs by the force that is directly applied on the mass, and the second type is ground disturbance; it occurs by the vibrations that come from the ground. These two types of vibrations can somehow be handled using passive and active suspension systems. However, at this point, performance and sustainability problems increase. The main reason of this situation is that; there is a trade-off principle between the designs of suspension elements that handle the vibrations mentioned above. If a suspension design that is stiff enough to handle direct disturbances is proposed, performance of the system on ground disturbances decreases. If a suspension design that is soft enough to suppress ground disturbances is proposed, performance of the system becomes unable to cope with direct disturbances. For having a brand new solution of this tradeoff, researchers have been focusing on the topic of obtaining negative-stiffness. In theory, when a spring that has positive stiffness is serially linked to another spring that has negative stiffness (for sure this is not possible in application), infinite stiffness occur against direct disturbances if and only if absolute values of stiffness parameters are equal. Moreover, if the spring that has positive stiffness is linked to the ground, its stiffness value can be adjust to get rid of ground disturbances, which means that the trade-off principle mentioned above vanishes and a vibration isolation system that is robust against both vibration types is designed [10]. At this point, hybrid levitation systems start to be useful. Because of a simple hybrid levitation system's structure, negative stiffness and zero-power features are both achievable [11].

There are many studies of realizing negative stiffness for 
hybrid electromagnets [2-5]. These advanced studies achieve negative stiffness meanwhile using zero-power control schemes at different DOF's. Therefore, zero-power control schemes (1. Realization of zero-power and negative stiffness, 2. Stiffness adjustment of zero power control [3]) are strictly coupled with obtaining negative stiffness in literature. In this work, a model matching $H \infty$ state feedback controller using linear matrix inequality optimization method is proposed for obtaining negative stiffness on direct disturbance case. In the simulation scenario, the applied force is measured by a sensor and then turned to voltage, after that, the effect of voltage, which is a controller input, on model matching error is minimized by decreasing $\|$ Gplant $\|\infty-\|$ Gmodel $\| \infty$ as much as possible, as if it is a disturbance attenuation problem.

\section{NEGATIVE STIFFNESS \& VIBRATION ISOLATION}

\section{A. Negative Stiffness}

Consider the system shown in Fig. 1 below. The spring hangs in a stretched position because of the mass. If an external downward force were applied to the mass, the spring would move downward. This is the definition of positive stiffness [12].

A hybrid electromagnet system can be controlled as if it is a spring which shows negative stiffness behavior. A system like this is shown in Fig 2. When external downward force is applied to the levitated mass, vertical position change is measured by a sensor, and electromagnets are driven based on a control law $[2-4,10]$.

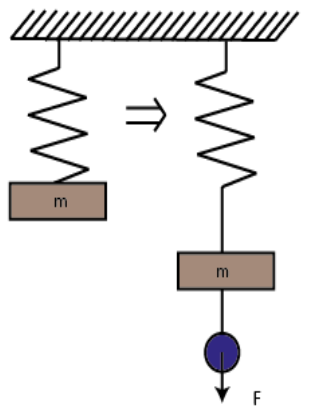

Fig. 1: Positive stiffness

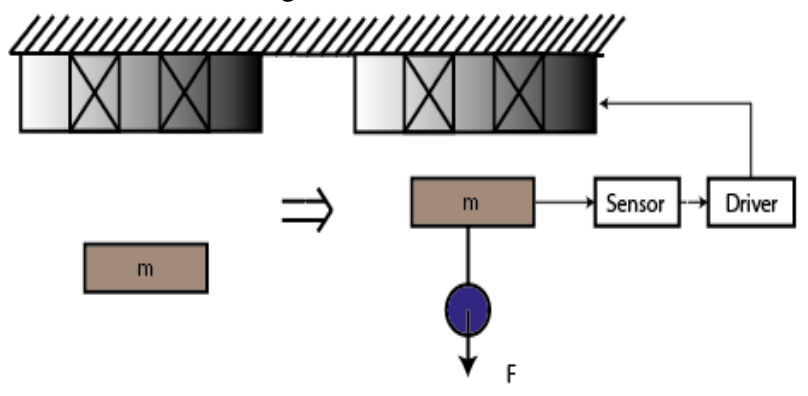

Fig. 2: Negative stiffness

\section{B. Vibration Isolation}

So far, the definition of negative stiffness and how to obtain it by using a hybrid electromagnetic system are explained. The question is, how negative stiffness can be used for vibration isolation purpose. This question is answered in the system shown in Fig 3 below. A spring that shows positive stiffness behavior is serially connected to a spring that shows negative stiffness behavior (in theory, of course) [2,3].

Equivalent spring coefficient is being equal to;

$$
k_{e}=\frac{k_{1} k_{2}}{k_{1}+k_{2}}
$$

For

$$
k_{1}=-k_{2}
$$

Equivalent spring coefficient becomes;

$$
k_{e}=\infty
$$

In Fig. 4, how to obtain infinite stiffness by using a hybrid electromagnetic system as a spring that shows negative stiffness behavior is explained [2, 3]. The hybrid electromagnetic system is serially connected to a spring that shows positive stiffness behavior.

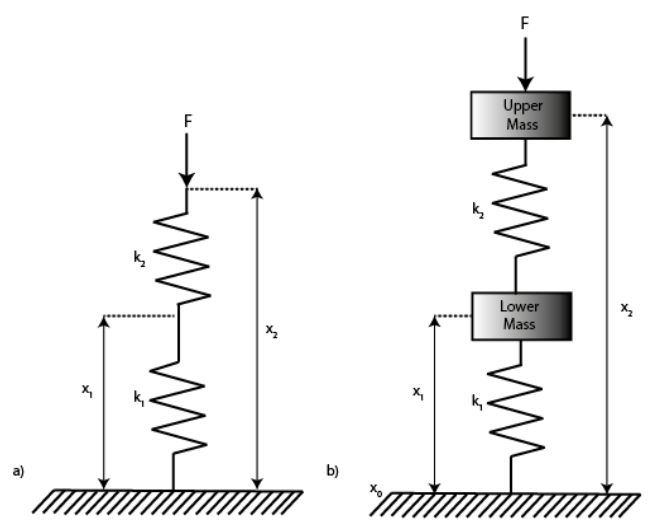

Fig. 3: Obtaining infinite stiffness

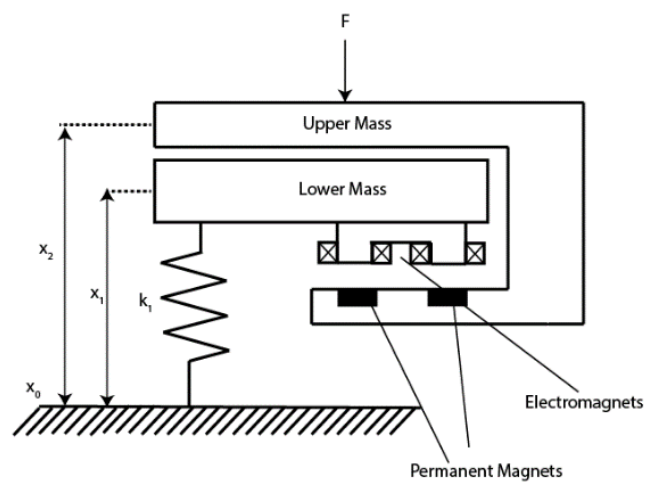

Fig. 4: Obtaining infinite stiffness with hybrid electromagnet

\section{Four Pole U-TyPe HybriD EleCtromagnet \& CONTROLLER DYNAMICS}

\section{A. Structure of Four Pole U-Type Hybrid Electromagnet}

The hybrid electromagnet contains four different poles joint together by an iron yoke. Each pole is controlled by a different external voltage source, and also, each pole has a different permanent magnet that create magnetic force. The structure of a simple hybrid electromagnet can be seen in Fig. 5 below 


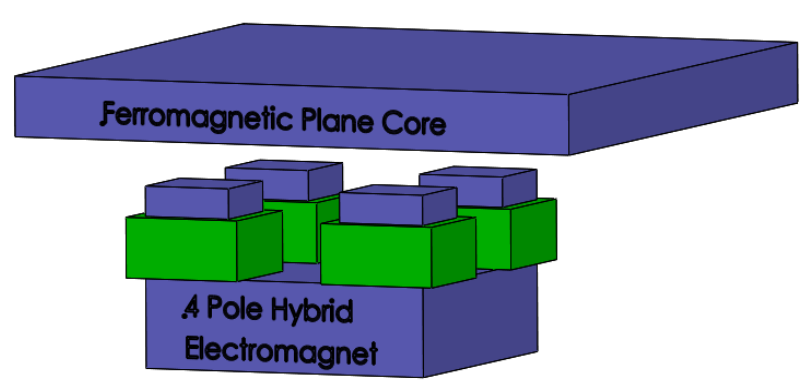

Fig. 5: Four pole u-type hybrid electromagnet

\section{B. Modeling Four Pole U-Type Hybrid Electromagnet}

In the model, it is assumed that eddy currents, flux leakage effects, fringing effects, magnetic resistance, magnetic saturation and hysteresis are negligible. The developed electromagnetic force for vertical direction is given below;

$$
F_{e}=k\left(\frac{i_{c}+I_{m}}{g_{e}+\frac{L_{m}}{\eta_{r}}}\right)
$$

$I_{c}$ is the coil current, $I_{m}$ current source equivalent of permanent magnet, $g_{e}$ is the equivalent gap clearance, $L_{m}$ is the total length of permanent magnets, $k$ is the configuration parameter of the hybrid electromagnet, $\eta_{r}$ is the relative permeability of the utilized permanent magnet.

As it can be seen from Eq. 4, the system exhibits nonlinear behavior. Linearization process is applied for $i_{z}=i_{z 0}$ and $z=z_{0}$ as follows;

$$
\begin{gathered}
K_{z}=\frac{\partial F_{e}}{\partial z}=2 k \frac{\left(i_{z}+I_{\mathrm{m}}\right)^{2}}{\left(z+\frac{L_{m}}{\eta_{r}}\right)^{3}} \\
K_{i}=\frac{\partial F_{e}}{\partial i_{z}}=2 k \frac{\left(i_{z}+I_{\mathrm{m}}\right)}{\left(z+\frac{L_{m}}{\eta_{r}}\right)^{2}} \\
F_{e}=K_{z} z+K_{i} i_{z}
\end{gathered}
$$

Four pole u-type hybrid electromagnet can control 3 degree of freedom by excitation of each one of the pole coils in an appropriate sequence. In this study, negative stiffness is created only for vertical direction. Thus, the governing dynamical equations of the hybrid electromagnet are derived only for vertical direction.

$$
\begin{gathered}
m \frac{d^{2} z}{d t^{2}}=F_{e}-m g-F_{d} \\
m \frac{d^{2} z}{d t^{2}}=K_{z} z+K_{i} i_{z}-m g-F_{d}
\end{gathered}
$$

$m$ is the mass of levitated object, $F_{d}$ is the external disturbance force (in this study, it is assumed that $F_{d}$ is assumed to be white noise), $g$ is the gravity acceleration. The electrical dynamics of the system is given below:

$$
\frac{d^{2} i_{z}}{d t^{2}}=-\frac{K_{z}}{K_{i}} \frac{d z}{d t}-\frac{R}{L} i_{z}+\frac{1}{L} V_{z}
$$

Therefore, the system's state-space representation (LTI system) can be written as follows;

$$
\begin{aligned}
& \left.\begin{array}{c}
\dot{z} \\
\ddot{z} \\
\ddot{i} \\
\dot{x}
\end{array}\right]=\underbrace{\left[\begin{array}{ccc}
0 & 1 & 0 \\
\frac{K_{z}}{m} & 0 & \frac{K_{i}}{m} \\
0 & -\frac{K_{z}}{K_{i}} & -\frac{R}{L}
\end{array}\right]}_{A}\left[\begin{array}{c}
z \\
\dot{z} \\
i_{z} \\
z
\end{array}\right]+\underbrace{\left[\begin{array}{ccc}
0 & 0 & 0 \\
-1 & 0 & -\frac{1}{m} \\
0 & \frac{1}{L} & 0
\end{array}\right]}_{B}\left[\begin{array}{c}
g \\
V \\
F_{d} \\
u
\end{array}\right] \\
& y=\underbrace{\left[\begin{array}{lll}
1 & 0 & 0 \\
0 & 1 & 0 \\
0 & 0 & 1
\end{array}\right]}_{C}\left[\begin{array}{c}
z \\
\dot{z} \\
i_{z}
\end{array}\right]+\underbrace{\left[\begin{array}{lll}
0 & 0 & 0 \\
0 & 0 & 0 \\
0 & 0 & 0
\end{array}\right]}_{D}\left[\begin{array}{c}
g \\
V \\
F_{d}
\end{array}\right]
\end{aligned}
$$

\section{Constructing Optimal CONTROL Structure of LTI MODEL}

Simple optimal controller scheme for disturbance attenuation problem can be seen in Fig. 6 below, and the mathematical model is given with Eq. (13), Eq. (14), and Eq. (15).

$$
\begin{array}{r}
\dot{x}=\underline{A} x+B_{\omega} \omega+B_{u} u \\
z=C_{z} x+C_{z \omega} \omega+D_{z u} u \\
y=C_{y} x+D_{y \omega} \omega
\end{array}
$$

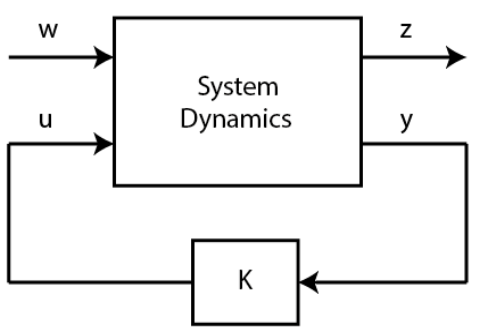

Fig. 6: Optimal control scheme

The goal here is to minimize the effect of $\omega$ on the magnitude of $z$ using feedback controller. To do this, the value given in Eq. (16) has to be minimized using a specific controller.

$$
\left\|G_{\text {plant }}\right\|_{\infty}-\left\|G_{\text {model }}\right\|_{\infty}<\gamma
$$




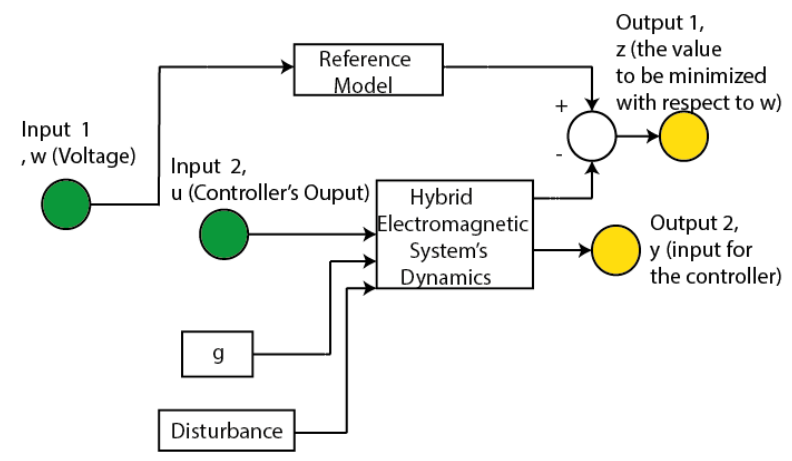

Fig. 7: Rearrangement of LTI system

KYP Lemma;

$$
\begin{aligned}
& {\left[\begin{array}{ccc}
A^{T} X+X A & X B & C^{T} \\
(X B)^{T} & -\gamma I & D^{T} \\
(-\gamma I)^{T} & D & -\gamma I
\end{array}\right]<0} \\
& X=X^{T}>0
\end{aligned}
$$

Following controller parameters are obtained;

$$
\begin{gathered}
C_{c}=\left(-F+D_{c} C_{y} Y\right) Y^{-1} Z^{-1} \\
B_{c}=G-X B_{u} D_{c} \\
A_{c}=\left[X\left(A+B_{u} D_{c} C_{y}-B_{u} C_{c} Z\right) Y+B_{c} C_{y} Y-L\right] Y^{-1} Z^{-1}
\end{gathered}
$$

where;

$$
\begin{gathered}
L=X\left[A+B_{u} D_{c} C_{y}-B_{u} C_{c} Z\right] Y+\left[B_{c} C_{y}-A_{c} Z\right] Y \\
F=-C_{c} Z Y \\
H=D_{c} \\
G=X B_{u} D_{c}+B_{c} \\
\underline{B}=\left[\begin{array}{ll}
B_{\omega} & B_{u}
\end{array}\right] \\
\underline{C}=\left[\begin{array}{l}
C_{z} \\
C_{y}
\end{array}\right] \\
\underline{D}=\left[\begin{array}{ll}
D_{z \omega} & D_{z u} \\
D_{y \omega} & D_{y u}
\end{array}\right]
\end{gathered}
$$

The system parameters given in Table I are obtained from a real experimental setup for comparing simulation results with experimental results in future studies.
TABLE I. SYSTEM PARAMETERS.

\begin{tabular}{|c|c|}
\hline Unit & Value \\
\hline $\mathrm{m}[\mathrm{kg}]$ & 16.35 \\
\hline$k\left[\mathrm{~N}^{2} / \mathrm{A}^{2}\right]$ & $1.04 \times 10^{-4}$ \\
\hline$I_{m}[\mathrm{~A}]$ & 13.83 \\
\hline$R[\Omega]$ & 1.018 \\
\hline$L[\mathrm{H}]$ & 0.0235 \\
\hline$K_{i}[\mathrm{~N} / \mathrm{A}]$ & 22.15 \\
\hline$K_{z}[\mathrm{~N} / \mathrm{m}]$ & 28058 \\
\hline
\end{tabular}

\section{SiMULATION RESULTS}

In Fig. 8, it can be seen that the behavior of hybrid electromagnetic system's dynamic output converges reference model's $\left(k_{r e f}=-5 \mathrm{~N} / \mathrm{m}\right)$ dynamic output, which means that control algorithm successfully works so far and steady-state error is in appropriate ranges.

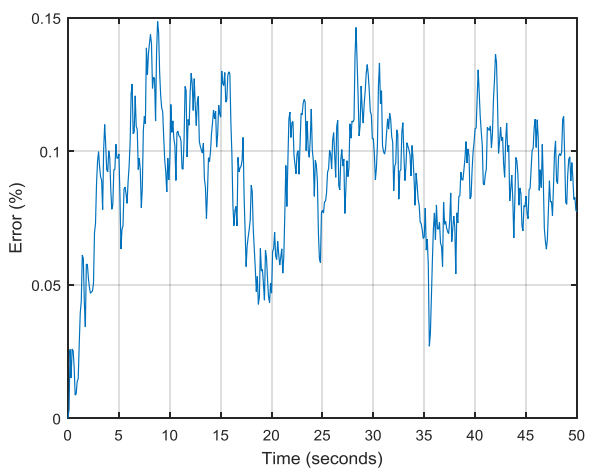

Fig. 8: Percentage error

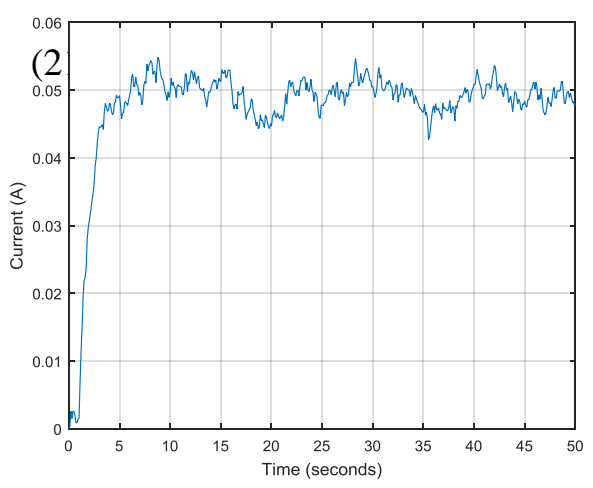

Fig. 9: Required current scheme

In Figure 9, it can be seen that even though optimal controller is not designed for zero-power control of the current, system's current performance locates in a range close to 0 value.

\section{CONCLUSION}

Designed optimal controller satisfies expected performance for $k_{r e f}=-5 \mathrm{~N} / \mathrm{m}$ stiffness, future work will include $H_{\infty}$ state feedback controller design using linear matrix inequality optimization method with an experimental setup, moreover, not only for model reference, but also zero-power control. 


\section{REFERENCES}

[1] Erkan, K., B. Acarkan, and T. Koseki. Zero-Power Levitation Control Design for a 4-Pole Electromagnet on the Basis of a Transfer Function Approach. in Electric Machines \& Drives Conference, 2007. IEMDC '07. IEEE International. 2007. http://dx.doi.org/10.1109/IEMDC.2007.383695

[2] Emdadul Hoque, M., et al., A six-axis hybrid vibration isolation system using active zero-power control supported by passive weight support mechanism. Journal of Sound and Vibration, 2010. 329(17): p. 34173430 . http://dx.doi.org/10.1016/j.jsv.2010.03.003

[3] Hoque, M.E., et al. A 3-DOF modular vibration isolation system using zero-power magnetic suspension with adjustable negative stiffness. in Advanced Motion Control, 2010 11th IEEE International Workshop on. 2010.

[4] Hoque, M.E., et al., A three-axis vibration isolation system using modified zero-power controller with parallel mechanism technique. Mechatronics, 2011. 21(6): p. 1055-1062. http://dx.doi.org/10.1016/j.mechatronics.2011.05.002

[5] Mizuno, T., et al., Vibration isolation system combining zero-power magnetic suspension with springs. Control Engineering Practice, 2007. 15(2): p. 187-196. http://dx.doi.org/10.1016/j.conengprac.2006.06.001

[6] Y. Yakushi, T.K., S. Sone, 3 degree-of-freedom zero power magnetic levitation control by a 4-pole type electromagnet, in International Power Electronics Conference2000: Tokyo, Japan.

[7] J. Liu, K.Y., T. Koseki, S. Sone. 3 degrees of freedom control of zeropower magnetic levitation for flexible transport system. in The 16th International Conference on Magnetically Levitated Systems and Linear Drives. 2000. Rio de Janeiro, Brazil.

[8] Mingda, Z., L. Xiaolong, and L. Shaoke. Research on the design of mechanical and electromagnetic composite vibration isolation system. in Prognostics and System Health Management Conference (PHM-2014 Hunan), 2014. 2014.

[9] Shahadat, M.M.Z., et al. On the simultaneous realization of virtually zero-power and zero-compliance controls. in Decision and Control (CDC), 2013 IEEE 52nd Annual Conference on. 2013.

[10] Hoque, M.E., et al., Development of a three-axis active vibration isolator using zero-power control. Mechatronics, IEEE/ASME Transactions on, 2006. 11(4): p. 462-470. http://dx.doi.org/10.1109/TMECH.2006.878536

[11] Mizuno, T., et al. Application of displacement cancellation control to vibration isolation system. in Control, Automation and Systems, 2007. ICCAS '07. International Conference on. 2007.

[12] Tao, Z., et al. A control strategy using negative stiffness for active vibration isolation. in Nano/Micro Engineered and Molecular Systems, 2008. NEMS 2008. 3rd IEEE International Conference on. 2008.

[13] Yiping, C. A Proof of the Discrete-Time KYP Lemma Using Semidefinite Programming Duality. in Control Conference, 2007. CCC 2007. Chinese. 2007. 